\title{
Investigação em serviços de saúde: alguns apontamentos históricos, conceituais e empíricos
}

\author{
Health services research: historical, conceptual, \\ and empirical highlights
}

Investigación en servicios de salud: algunos
apuntes históricos, conceptuales y empíricos

Mônica Martins 1

Margareth Crisóstomo Portela 1

Marina Ferreira de Noronha 1

doi: 10.1590/0102-311X00006720

\section{Introdução}

A investigação em serviços de saúde ou pesquisa em (ou sobre) serviços de saúde - traduções adotadas no Brasil para pesquisa em serviços de saúde (health services research - HSR) 1,2,3,4 - é um campo multidisciplinar que trata de temas relacionados ao desempenho de sistemas e serviços de saúde, acesso e utilização de serviços, qualidade do cuidado e custos. Examinados à luz de arcabouços teórico-conceitual e metodológico oriundos de disciplinas das ciências sociais, da saúde e exatas, essas temáticas articulam abordagens biomédica, epidemiológica, econômica, estatística, comportamental, social, organizacional e política 5,6,7,8. Essa variedade de olhares e abordagens é sua riqueza, pois permite aproximação mais nítida de problemas complexos relacionados à saúde de indivíduos e população e à prestação de cuidado em saúde.

Considerando-se o conhecimento acumulado ao longo de décadas, este artigo apresenta uma visão geral e pretende servir como um convite a uma revisita à literatura sobre o campo. A experiência acumulada é instigante, aporta contribuições teórico-metodológica e prática, com questões que evoluíram no tempo, acompanhando o aumento da complexidade dos sistemas de cuidados de saúde. Espera-se inspirar pesquisadores a examinar, aprofundar e produzir evidências que subsidiem, no presente, a sociedade e tomadores de decisão o esboço de uma agenda para garantir acesso, qualidade e equidade no Sistema Único de Saúde (SUS). Nessa agenda, a sustentabilidade de sistemas de saúde de qualidade é colocada como um desafio global.

\section{Origem e evolução conceitual do campo}

O termo HSR foi adotado em 1960, para substituir o nome da seção de estudos das Instalações Hospitalares e Médicas (Hospital and Medical Facilities) do Institutos Nacionais de Saúde (National Institutes of Health - NIH) nos Estados Unidos 9,10. A partir daí, foram muitos os desdobramentos e significativo o desenvolvimento do campo. Em 1962, o canadense Kerr White, liderança importante no campo, assumiu a direção da nova seção de investigação em serviços de saúde do NIH. Nos anos seguintes, foram instituídas linhas de financiamento de estudos e comissionados artigos com foco nas diferentes facetas e questões relevantes do campo, publicados em 1966, no volume 44 da Milbank
1 Escola Nacional de Saúde Pública Sergio Arouca, Fundação Oswaldo Cruz Rio de Janeiro, Brasil.

Correspondência M. Martins Departamento de Administração e Planejamento em Saúde, Escola Nacional de Saúde Pública Sergio Arouca, Fundação Oswaldo Cruz. Rua Leopoldo Bulhões 1480, 70 andar, Rio de Janeiro, RJ 21041-210, Brasil. martins@ensp.fiocruz.br 
Memorial Fund Quarterly. Também foram criados centros dedicados à investigação em serviços de saúde e periódicos científicos específicos, como Health Services Research e Medical Care. Em 1968, foi criado nos Estados Unidos o Centro Nacional de Pesquisas em Serviços de Saúde (National Center for Health Services Research) que, extinto em 1989, deu lugar à Agency for Health Care Policy and Research, renomeada em 1999 Agency for Health Research and Quality (AHRQ), ainda hoje referência fundamental na área 10. Ainda em 1968, foi publicado, na Milbank Memorial Fund Quarterly, um artigo importante na delimitação do campo, relativo à experiência na Grã-Bretanha 11.

A definição do campo da investigação em serviços de saúde foi modificada e ampliada ao longo do tempo, adquirindo novos contornos e interseção com campos correlatos, como o cuidado clínico ou política de saúde 12. Igualmente, outras denominações ou recortes, como avaliação do cuidado, dos serviços, da qualidade e dos sistemas de saúde ou mesmo avaliação econômica e tecnológica se debruçam sobre problemas e desafios, amplos ou específicos, relativos à produção e prestação do cuidado em saúde.

Em 1979, o Instituto de Medicina estadunidense (IoM) estadunidense definiu a investigação em serviços de saúde como pesquisa para produzir conhecimento sobre a estrutura, processos e efeitos de serviços de saúde 12. Em 1995, a redefiniu como campo multidisciplinar de pesquisa, básica e aplicada, que examina o uso, custos, qualidade, acessibilidade, provisão, organização, financiamento e resultados dos serviços de cuidado de saúde para aumentar o conhecimento da estrutura, processos e efeitos dos serviços de saúde para indivíduos e populações 12,13.

Kerr White 14 já levantava, em 1961, questões acerca da efetividade da provisão de cuidados médicos à população, frente ao elevado investimento em pesquisa, bem como à avaliação pelos consumidores da quantidade, qualidade e distribuição dos cuidados disponíveis. Ele alternou entre os termos health services research e health care research, e chegou a assumir o último como o mais abrangente 9,15 . Optando pelo primeiro termo em artigo publicado em 2000, entretanto, sugeriu a investigação em serviços de saúde como campo de estudo dos resultados, benefícios, riscos e custos relativos das intervenções em saúde voltadas para indivíduos e populações 10. Também sublinhou que as análises são de base populacional, sendo assim dependentes deste tipo de informação e orientadas pela ideia de comparação 16 .

Numa concepção ampla, a investigação em serviços de saúde trata de macrodeterminantes, elementos constituintes do sistema de saúde (legislação, recursos e organização), do processo desde o surgimento de uma necessidade de saúde, acesso e uso dos serviços até o resultado - modificação desta necessidade 17, incluindo a qualidade do cuidado em todas as suas dimensões 18,19. Essa assertiva se confirma na definição adotada pela AHRQ nos anos 2000, que compreende a investigação em serviços de saúde como campo multidisciplinar de pesquisa dedicado ao estudo de como os fatores sociais, sistemas de financiamento, estruturas e processos organizacionais, tecnologias em saúde e comportamentos individuais afetam o acesso ao cuidado em saúde, a qualidade e os custos, e a saúde e bem-estar das pessoas 12,13,20. As fronteiras são muitas vezes tênues, e mesmo difíceis de distinguir, justificadas talvez pela formação do autor que os emprega ou prefere, mas também pelo reconhecimento da complexidade dos fenômenos envolvidos em cada âmbito de análise - de indivíduos a populações 12,20. A expectativa é prover dados, evidência e instrumentos para tornar os cuidados de saúde economicamente viáveis, seguros, efetivos, equânimes, acessíveis e centrados nos pacientes 13,21.

Enfim, a amplitude temática, acompanhada pela variedade conceitual-metodológica, aporta polissemia terminológica, alguma imprecisão operacional e mesmo confusão entre pesquisadores formados em distintas disciplinas do campo 6,7,22. Essa diversidade de formação requer harmonização entre teorias econômicas, sociológicas, psicológicas e políticas e na aplicação de métodos de pesquisa, buscando garantir a conjugação, de fato, das distintas disciplinas acadêmicas na avaliação de determinada questão ${ }^{6}$. Assim, a garantia do caráter multidisciplinar está sempre em construção, requerendo contínuo aprendizado e melhoria.

Desse introito depreende-se que a investigação em serviços de saúde incorpora uma ampla gama de questões sobre a estrutura e desempenho dos serviços e do sistema de saúde, necessidade de saúde, demanda, acesso e uso dos serviços e a qualidade do cuidado prestado. Inserida nesse campo emergem questões centrais referentes ao acesso, continuidade, efetividade, eficiência, equidade, eficácia, adequação, oportunidade do cuidado, segurança do paciente e centralidade do cuidado no paciente. Embora imbricadas, algumas dessas questões ganharam destaque na agenda sanitária, decorrente de 
fatores contextuais do momento e lugar, além das características dos sistemas de saúde. Contudo, algumas mantiveram-se perenes na agenda, como a efetividade, eficiência e equidade 8,20,23,24,25,26.

\section{Serviços de saúde como objeto: enfoques, pesquisadores e estudos emblemáticos}

Estudos seminais focando temas pertinentes ao campo da investigação em serviços de saúde foram produzidos antes dos anos 1960, como os desenvolvidos por Florence Nightingale, Ernerst Codman ou Alison Glover 10,27. A partir da formalização do campo nos anos 1960, merecem destaque eminentes pesquisadores, contemporâneos de White 14,15,16, que estabeleceram bases fundacionais de áreas que compõem e se articulam no campo. Entre tais pesquisadores, listam-se Uwe Reinhardt (economia da saúde) 28, Avedis Donabedian (qualidade do cuidado) 18,29,30, Archie Cochrane (eficácia, efetividade e eficiência) 31, Milton Roemer (Roemer's law e uso hospitalar) 32, Julian Tudor-Hart (inverse care law) 33, Walter Holland (avaliação do cuidado em saúde) 25, Brian Abel-Smith (financiamento) 34, John Eisenberg (decisão médica) 35, Ronald Andersen (acesso e uso) 36, Harold Luft (volume e qualidade) 37, Michael Drummond (avaliação econômica) 38, David Banta (avaliação tecnológica) 39, Gavin Mooney (equidade) 40, Mark Chassin (qualidade, accountability) 41,42, John Wenneberg (variação geográfica e injustificada da prática) 43,44 e Robert Brook (qualidade e adequação) 8,45. Oriundas dessa vasta produção, duas linhagens de lideranças foram estabelecidas, uma de norte-americanos e outra de ingleses.

Os últimos 60 anos foram marcados pelo aumento da demanda, complexidade e custos dos serviços de saúde e crescente conscientização da limitação dos recursos disponíveis nos diversos sistemas de saúde do mundo. Tal contexto constituiu-se em fator desencadeador da necessidade de informação e pesquisa sobre a qualidade, disponibilidade e custos dos serviços de saúde 25,26,46,47,48. Partindo da realidade norte-americana, Wyzewianski 47 descreveu os anos 1960 e 1970 como o período no qual a qualidade emergiu como questão relevante e a década de 1980 como o período da "consciência pública" da qualidade.

Nos Estados Unidos e Canadá, o foco na eficiência e efetividade para garantir a qualidade foi continuadamente extenso 47; na Inglaterra a atenção esteve voltada para a análise do desempenho dos serviços de saúde, na qual a equidade constituiu, também, eixo de preocupação 26,49,50. Apesar da predominância de estudos preocupados com a utilização de recursos limitados, observou-se na literatura a ampliação do escopo, agregando outros pontos para além da eficiência e efetividade, como acesso, adequação e equidade, expressos claramente no relevo adquirido pela avaliação de qualidade 18,29,30,51. Essa, majoritariamente escrutinizada com base nos aportes de Donabedian 29,30,52, nas décadas de 1960 e 1970, abordava sobretudo o processo de cuidado. Paulatinamente, nos anos seguintes, ampliou-se o interesse por medidas do resultado do cuidado - efeitos do cuidado sobre o bem-estar dos pacientes 53 , mesmo considerando problemas na validade causal e atribuível 30 . Concomitante à produção de estudos, foram diversas as tentativas de contenção dos custos na prestação de cuidados de saúde, paralelamente ao monitoramento de eventual piora no desempenho dos serviços de saúde 26,46 .

A evolução e escopo da investigação em serviços de saúde nas suas três primeiras décadas podem claramente ser dimensionados na Antologia organizada por White et al. 54. Partindo da consulta a 750 especialistas com experiência na área, dos quais 230 indicaram até 10 referências, foram elencados 100 estudos relevantes para a consolidação do campo, dentre os quais foram inseridos artigos de pesquisadores antes citados. Os 100 estudos selecionados focalizam: equidade, acesso, qualidade e custos do cuidado em saúde, questões metodológicas e evidências sobre variação no cuidado, eficácia e efetividade e resultado sobre a saúde do indivíduo e população.

Nos anos 1990, com o emprego de grandes bases de dados populacionais e administrativos, ganharam importância estudos sobre variações nas práticas de cuidado e seus efeitos no padrão de utilização de serviços e nos resultados 37,41,44. Análises de pequenas áreas foram úteis para a detecção de variações não explicadas pelo perfil da demanda e atribuíveis a estilos diferenciados de prática 44. Passaram, também, a estudar variações nos resultados do cuidado (outcomes research) relacionadas a práticas/intervenções diversas, provendo evidências acerca da adequação do cuidado para diagnóstico e tratamento de pacientes com condições clínicas definidas, assim como dos riscos intrínsecos $35,44,55$. 
Documentos publicados pelo IoM nos anos 2000, especialmente To Err Is Human: Building a Safer Health System 56 e Crossing the Quality Chasm: a New Health System for the 21st Century 19, colocaram a segurança e a centralidade do cuidado no paciente como dimensões da qualidade do cuidado de saúde a serem perseguidas, além da efetividade, eficiência, equidade e oportunidade. Desde então, tais dimensões são centrais na agenda da investigação dos serviços de saúde em vários países.

Ainda, frente à forte preocupação com a elevação dos custos dos cuidados e a necessidade de controle, estabeleceu-se sinergia entre avaliação da qualidade e prestação de contas, em alinhamento ao que Relman 46 denominou "terceira revolução" no sistema de prestação do cuidado em saúde. Preocupações com custo e qualidade também desembocaram no emprego de mecanismos de pagamento por desempenho em diversos sistemas de saúde 57 , cuja efetividade tem sido objeto de avaliação.

Nos últimos 10 anos, a implementação de abordagem científica à concepção, implementação e avaliação de intervenções para a melhoria da qualidade do cuidado, com vistas à produção de conhecimento generalizável, valendo-se de bases teóricas, acúmulo de experiências locais e valorização dos aspectos contextuais, tem-se inspirado no e se imbricado ao campo da investigação em serviços de saúde 58,59,60,61. O foco no cuidado centrado no paciente ampliou-se, com a valorização da experiência do paciente no processo de cuidado de saúde 23 e do compartilhamento de decisões entre profissionais de saúde e pacientes. A coordenação do cuidado continua sendo um ponto desafiador na agenda, colocando-se a necessidade de aprimoramento de recursos organizacionais e tecnológicos, mas, especialmente, de entendimento e transformação de fenômenos sociais pertinentes à provisão de serviços de saúde. No centro das prioridades há questões relevantes para a sustentabilidade dos sistemas de saúde, como custos crescentes, uso excessivo (overuse) ou cuidado de baixo valor/beneficio (low-value care) associados ao desperdício de recursos, ocorrência de eventos adversos e consequentemente maior necessidade de prestação de contas (accountability) 41,42,62,63,64,65,66,67,68.

Ademais, persistem questões metodológicas desafiadoras, como a definição parcimoniosa de medidas validadas para a avaliação de aspectos do cuidado, o aperfeiçoamento de processos de coleta e interpretação de dados 69, o emprego promissor de Big data, maior incorporação da pesquisa qualitativa junto à quantitativa e, especialmente, a lacuna entre as evidências produzidas pelos estudos e a translação destas para o cuidado prestado 42,44,70,71. Concernente a essa lacuna, Kupersmith \& Eisen 71 destacaram a ausência de mecanismo efetivo e regular de articulação e coordenação entre pesquisadores e tomadores de decisão política, gestores e lideranças clínicas, constatação que surpreende, mas não é nova $27,43,70$.

Em termos gerais, o campo, de grande pungência, se apropria amplamente de desenhos de estudo experimentais, quase experimentais e observacionais, bases de dados primárias e secundárias e de técnicas estatísticas descritivas e analíticas, além de técnicas de síntese, seja da literatura ou de dados, como as análises de custo-efetividade 13,61. Todavia, coloca-se a importância da pesquisa qualitativa, com vistas à explicação de como e por que a coisas ocorrem 13 .

\section{Investigação ou avaliação de serviços de saúde - contexto brasileiro}

Embora no cenário internacional o campo e o termo investigação em serviços de saúde tenham ganhado vulto e ações concretas, produção de estudos, criação de agências governamentais de monitoramento e financiamento de pesquisas, associações, periódicos científicos e espaço nas universidades, no contexto brasileiro esta plêiade temática foi ancorada em distintas áreas na Saúde Coletiva, em particular no Planejamento e Epidemiologia. Comparativamente, o volume e escopo da produção nacional são menores. Ademais, os estudos tratando de temas como necessidade em saúde, demanda, oferta, financiamento, uso, qualidade e resultados dos serviços de saúde são reconhecidos preferencialmente no termo "avaliação de serviços de saúde" ou "epidemiologia e serviços de saúde" ou ainda "economia da saúde". Linhas de pesquisa sobre avaliação de serviços de saúde, avaliação do cuidado em saúde, desempenho e qualidade, por exemplo, utilizadas por grupos de pesquisa brasileiros, embutem conceitos multidimensionais que misturam ou tangenciam o que foi definido como investigação em serviços de saúde.

Marcos explícitos da incorporação do campo no país podem ser localizados entre a segunda metade dos anos 1980 e primeira dos anos 1990, quando também se vivia o processo de redemocratização, 
promulgação da nova Constituição e instituição do SUS. Em 1988, foi realizado, na Fundação Oswaldo Cruz, o Seminário Internacional de Metodologia de Avaliação de Serviços de Saúde (SIMASS), organizado por Claudia Travassos e Leticia Krauss, com o apoio da Organização Pan-Americana da Saúde (OPAS), com o objetivo de ampliar a capacidade técnica na área. No início da década de 1990, um minicurso promovido pela Fundação Getulio Vargas, coordenado por Ana Malik, trouxe ao Brasil o principal protagonista da área de avaliação da qualidade do cuidado, Avedis Donabedian, o que foi uma oportunidade única para pesquisadores interessados. Em paralelo e com muitas interfaces com a "Avaliação de Serviços de Saúde", também emergiam no país as áreas de "Economia da Saúde" e da "Avaliação de Tecnologias em Saúde", com alguns estudos pioneiros sendo realizados. A OPAS e OMS foram importantes fomentadores de iniciativas, entre as quais também destaca-se o "Curso de Investigação em Serviços de Saúde", originalmente desenvolvido pelo International Development Research Centre. O curso focava a capacitação de profissionais nos serviços de saúde, sendo oferecido pela Fundação Oswaldo Cruz, com o envolvimento de um conjunto de pesquisadores ${ }^{2}$. Duas perspectivas foram colocadas em relação ao campo, apontando tanto para o seu desenvolvimento na área acadêmica, via cursos de mestrado e doutorado, com o emprego rigoroso do método científico e técnicas sofisticadas, como a sua aplicação na solução de problemas no âmbito dos serviços de saúde. Em 1994, constituiu-se na América Latina a Rede de Investigação em Sistemas e Serviços de Saúde do Cone Sul (RED ISS), uma rede colaborativa com ações educativas para incrementar a pesquisa, capacitação e disseminação sobre os sistemas e serviços de saúde 72 . A rede sofreu alguma descontinuidade e redução de ações a partir de 2010.

Da produção nacional anterior à década de 1990, dois artigos foram incluídos na antologia organizada por White et al. 54, ambos focando o padrão de uso de cesarianas no Brasil 73,74, provavelmente inspirados no estudo pioneiro de Carlos Gentile de Mello 75 sobre a epidemiologia da cesariana em 1971.

Nos periódicos científicos da área da Saúde Coletiva, diversos artigos pertinentes à investigação em serviços de saúde têm sido publicados. Foram ainda publicados ao menos cinco números especiais que expressam como as temáticas arroladas vêm sendo trabalhadas no país. O primeiro, na Divulgação em Saúde para Debate, em 1991, continha artigos teóricos sobre avaliação da qualidade dos serviços 76, avaliação econômica 38 e avaliação tecnológica 39 , elaborados por pesquisadores estrangeiros de destaque nestas áreas, além de artigos sobre pesquisas em curso naquele momento. O número especial foi fruto do SIMASS, anteriormente referido. A disseminação em português dos artigos elaborados pelos convidados estrangeiros 38,39,76 representou um marco, sendo os mesmos muito referidos em publicações nacionais.

Em 1996, Cadernos de Saúde Pública publicou o Suplemento intitulado Epidemiologia e Avaliação de Serviços de Saúde, em que reconhecia a última como uma "nova área inter ou transdisciplinar" 77 e identificava uma agenda de pesquisa 78 . Nesse mesmo periódico, em 2004, outro número temático debruçou-se sobre Investigação e Avaliação em Serviços de Saúde 79, reconhecendo, passados 16 anos do seminário internacional, a persistência de lacunas conceituais e metodológicas nos estudos sobre sistemas, serviços e cuidado em saúde. Nesse número, um artigo de Novaes 4 apresentou um valioso panorama sobre a pesquisa em serviços de saúde nos contextos internacional e nacional, cujo debate alinhavou questões cruciais para a agenda nacional naquele momento, ainda pertinente, embora mais fragilizada, considerando a conjuntura atual da ciência e tecnologia e do SUS.

Os dois outros números temáticos aqui destacados foram publicados, respectivamente, na Divulgação em Saúde para Debate, em 2007, e na Revista Saúde em Debate, em 2017. O primeiro focou a Economia da Saúde, voltando-se para a divulgação da área e estímulo à articulação entre a pesquisa científica e a gestão dos serviços de saúde em sistemas universais, integrais e equitativos 80 . O outro, centrou na prática avaliativa e na influência de avaliações e dos sistemas de monitoramento para subsidiar projetos, programas e políticas de saúde 81 .

Alguns dos eventos e produção científica anteriormente destacados talvez ajudem a compreender as razões da supremacia do termo "Avaliação de Serviços de Saúde" sobre "Investigação em Serviços de Saúde", e ambiguidades na configuração do campo no contexto maior da Saúde Coletiva com seus pilares clássicos - Políticas, Planejamento e Gestão, Epidemiologia, e Saúde e Sociedade. A "Economia da Saúde", "Avaliação de Tecnologias em Saúde" e "Avaliação de Programas" trilharam caminhos próprios, com relevantes interseções. 
Estudos sobre o desempenho de sistemas de saúde, avaliação dos serviços de saúde e qualidade do cuidado no Brasil ganharam espaço na academia, em projetos de pesquisa, dissertações, teses e artigos. Ainda que com imperfeições e limites, informações oriundas de base de dados secundários, inquéritos populacionais 82 e pesquisas oficiais de abrangência nacional têm permitido análises sobre a distribuição da oferta e padrão de uso de serviços de saúde, variações nos processos e resultados do cuidado de saúde, incluindo a desigualdade, mortalidade hospitalar, financiamento e custos. Especialmente, três Suplementos Saúde da Pesquisa Nacional por Amostra de Domicílios do Instituto Brasileiro de Geografia e Estatística 82,83 geraram números temáticos da Ciência er Saúde Coletiva publicados em 2002, 2006 e 2011 83. Há uma diversidade de publicações relativas a diferentes condições de saúde e desigualdades sociais no estado de saúde. Destaca-se aqui o Projeto de Avaliação de Desempenho de Sistemas de Saúde (PROADESS), ainda ativo, cuja matriz de avaliação incorpora subdimensões do desempenho dos serviços de saúde como efetividade e acesso, e monitora um conjunto de indicadores destas subdimensões 84 .

Nas últimas duas décadas, vale apontar estudos de abrangência nacional demandados pelo Ministério da Saúde, conduzidos pela academia, que obtiveram dados primários relativos ao uso e à qualidade do cuidado. Citam-se aqui a Pesquisa Nacional de Saúde Bucal (2003 e 2010), a pesquisa Nascer no Brasil (2011-2012) e a Pesquisa Nacional de Saúde (2013). O Programa Nacional de Melhoria do Acesso e da Qualidade da Atenção Básica, também envolvendo pesquisadores de diferentes instituições, foi concebido com o propósito de avaliar e subsidiar melhorias na atenção básica no país. Apesar da adesão voluntária ao programa repercutir na representatividade dos dados, foi uma iniciativa relevante, que hoje parece condenada à descontinuidade.

Atividades desenvolvidas para avaliar os serviços de saúde no Brasil pelos organismos responsáveis têm se restringido, majoritariamente, a aspectos quantitativos, voltados para a mensuração de indicadores pactuados e de produtividade que media o repasse de recursos. Não há avaliações rotineiras e sistemáticas dos serviços prestados, o que resulta em obstáculos para o planejamento e gestão dos serviços de saúde. Essa carência de instrumentos contínuos de monitoramento e avaliação dificulta o acompanhamento das políticas, bem como a implementação de ações para a correção de barreiras de acesso e desigualdade no uso, na adequação, segurança e efetividade do cuidado prestado. Considerando as lacunas, avanços e descontinuidades, parece importante ratificar a necessidade, que persiste no país, de investimento na formação e aplicação de teorias e métodos do campo da investigação em serviços de saúde, reconhecendo que, como campo multidisciplinar, ainda padece de certa fragmentação entre o tripé da Saúde Coletiva, com alguma perda de identidade. Contudo, a relevância temática é inequívoca, requerendo contínua parceria entre academia, gestores, profissionais, usuários, sociedade e instancias governamentais.

\section{Considerações finais}

Este artigo oferece uma visão geral da investigação em serviços de saúde, mas a partir do olhar parcial moldado pelas leituras e trajetórias das autoras. Ao recontar a história e abordar a produção no campo, este convida pesquisadores experientes e iniciantes a lerem ou revisitarem os seus clássicos, e recoloca a necessidade de perseguir a uma maior apropriação de teorias e métodos pertinentes e atentar para velhos e novos desafios temáticos.

A investigação em serviços de saúde (health services research) é um campo multidisciplinar que trata de temas relacionados ao desempenho de sistemas e serviços de saúde, acesso e utilização de serviços, qualidade do cuidado e custos. Em geral, a avaliação dos serviços de saúde e especificamente da qualidade do cuidado cresceu nas últimas décadas. No Brasil, o campo da investigação em serviços de saúde não ganhou essa identidade, sendo mais frequentemente incorporada a denominação Avaliação de Serviços de Saúde ou Epidemiologia e Serviços de Saúde, talvez refletindo ambiguidades da Saúde Coletiva. Aspectos metodológicos e temas são continuamente tratados disciplinarmente, sendo o uso de abordagens multidisciplinares e métodos mistos em estudos voltados para os serviços e sistemas de saúde ainda incipiente e passível de aprimoramento e consolidação. Ademais, os contornos de delimitação da avaliação em serviços de saúde e avaliação de programas, conceitual e metodologicamente de bases distintas, terminam às vezes por se mesclarem. 
No contexto internacional, importa assinalar que existem lacunas a serem preenchidas no campo. Ainda que o volume de estudos seja considerável, resultados obtidos são frequentemente incomparáveis em função de recortes metodológicos diversos e (in)sucessos associados ao contexto de implementação. Assim, algumas questões podem ser respondidas por recortes metodológicos específicos, mas com a preocupação da comparabilidade e compartilhamento de experiências, com vistas à construção de conhecimento científico que subsidie a melhoria do desempenho dos sistemas e serviços de saúde. A aplicação de evidências nesse sentido ainda tem sido pequena em diversos países, apesar de algum acúmulo.

No Brasil, ainda detemos um conjunto de experiências isoladas e marcadas pela sazonalidade. Há maior desenvoltura no âmbito acadêmico. Carecemos na área da saúde, como em outros setores, no nível governamental, de uma tradição na avaliação dos resultados alcançados, dada a sua utilidade para a prestação de contas junto à sociedade acerca da qualidade do cuidado prestado no âmbito público e privado. Como atividade intrínseca de gestão e planejamento do sistema de saúde, o exposto antes ilustra a fragilidade das nossas atividades no campo da avaliação dos serviços de saúde, marcada pela descontinuidade e inexistência de um processo sistemático de acompanhamento do cuidado prestado.

Embora tenha havido muito acúmulo ao longo de décadas, "velhos" problemas/questões permanecem: alto custo; variações na prática devido ao uso insuficiente, inadequado ou excessivo; incorporação de tecnologias; (in)equidades nos serviços de saúde (oferta, acesso, uso e efetividade dos cuidados de saúde); e na alocação de recursos, organização e coordenação do cuidado e emergência de novas necessidades de saúde. Paralelamente, novos temas estão em pauta, alguns com nova roupagem, como ciência da melhoria, translação do conhecimento, cuidado seguro e centrado no paciente, preferência do paciente e tomada de decisão compartilhada, cuidado de baixo valor/benefício, desperdício de recursos e sustentabilidade dos sistemas de saúde. Os problemas antigos e novos, os olhares sobre novas facetas dos serviços e mudanças nas políticas públicas e de proteção social compõem um contexto cada vez mais complexo, resultando em novos desafios para o desenvolvimento de investigações em serviços de saúde.

\section{Colaboradores}

M. Martins, M. C. Portela e M. F. Noronha contribuíram na concepção, revisão da literatura, redação e revisão crítica do artigo.

\section{Informações adicionais}

ORCID: Mônica Martins (0000-0002-9962-0618); Margareth Crisóstomo Portela (0000-0002-98589276); Marina Ferreira de Noronha (0000-00022033-5532).

\section{Agradecimentos}

À Claudia Travassos, pesquisadora emérita da Fundação Oswaldo Cruz, cuja apresentação e vídeo (disponível em https://portal.fiocruz.br/video/ investigacao-em-servicos-de-saude-claudia-maria-travassos) sobre o tema inspiraram a concepção deste artigo. Ao Conselho Nacional de Desenvolvimento Científico e Tecnológico (CNPq): M. M. e M. C. P. são bolsistas de produtividade. 


\section{Referências}

1. Rodrigues Filho J. Pesquisa em serviços de saúde: uma necessidade urgente. Cad Saúde Pública 1989; 5:7-16.

2. Carvalheiro JR. Investigação em serviços de saúde: qual é o seu problema? Saúde Soc 1994; 3:64-111.

3. Teixeira CF. Investigação em sistemas e serviços de saúde: novos problemas e objetos, abordagens e estratégias. Saúde Soc 1997; 6:11-24.

4. Novaes HMD. Pesquisa em, sobre e para os serviços de saúde: panorama internacional e questões para a pesquisa em saúde no Brasil. Cad Saúde Pública 2004; 20 Suppl 2:S147-57.

5. Forrest CB, Martin DP, Holve E, Millman A. Health services research doctoral core competencies. BMC Health Services Res 2009; 9:10712.

6. Dowd BE. Separated at birth: statisticians, social scientists, and causality in health services research. Health Serv Res 2011; 46:397-420.

7. Maciejewski ML, Weaver EM, Hebert PL. Synonyms in health services research methodology. Med Care Res Rev 2011; 68:156-76.

8. Brook RH. Exploiting the knowledge base of health services research. In: Brook RH, editor. Redefining health care systems. Santa Monica: RAND Corporation; 2015. p. 5-30.

9. White K. Health care research: old wine in new bottles. Speech presented in the University of Virginia's History of the Health Sciences Lecture Series on September 24, 1992. In: Virginia University. The Kerr White Health Care Collection. http://historical.hsl.virginia.edu/ kerr/moreinfo.cfm.html (acessado em 14/ Dez/2019).

10. McCarthy T, White KL. Origins of health services research. Health Serv Res 2000; 35:37587.

11. Bierman P, Connors EJ, Flook E, Huntley RR, Carthy TM, Sanazaro PJ. Health services research in Great Britain. Milbank Mem Fund Q 1968; 46:9-102.

12. Lohr KN, Steinwachs DM. Health services research: an evolving definition of the field. Health Serv Res 2002; 37:15-8.

13. Shi L. Health services research methods. 3rd Ed. Boston: Cengage; 2019.

14. White KL, Williams F, Greenberg BG. Ecology of medical care. N Engl J Med 1961; 285:88392.

15. White K. The ecology of medical care: origins and implications for population-based healthcare research. Health Services Res 1997; 32:1121.

16. University of Virginia. The Kerr White health care collection, 2006. http://historical.hsl.virginia.edu/kerr/hsr.cfm.html (acessado em 09/ Dez/2019).

17. Donabedian A. Aspects of medical care administration: specifying requirements for health care. Cambridge: Harvard University Press; 1973.

18. Donabedian A. The seven pillars of quality. Arch Pathol Lab Med 1990; 114:1115-8.
19. Institute of Medicine; Committee on Quality of Health Care in America. Improving the 21 st century health care system. Crossing the quality chasm: a new health system for the 21 st century. Washington DC: National Academy of Sciences; 2001.

20. Begley CE, Lairson DR, Morgan RO, Rowan PJ, Balkrishnan R. Evaluating the healhtcare system: effectiveness, efficiency, and equity. 4th Ed. Chicago: Heath Administration Press; 2013.

21. Agency for Healthcare Research and Quality. Introduction. An organizational guide to build Health Services Research capacity. https:// www.ahrq.gov/funding/training-grants/ hsrguide/hsrguide.html (acessado em 27/ Dez/2019)

22. Maciejewski ML, Diehr P, Smith MA, Hebert P. Common methodological terms in health services research and their symptoms. Med Care 2002; 40:477-84.

23. Berwick DM, Nolan TW, Whittington J. The triple aim: care, health, and cost. Health Aff (Millwood) 2008; 27:759-69.

24. Aday LA, Begley CE, Lairson DR, Balkrishnan R. Evaluating the medical care system effectiveness, efficiency, and equity. Ann Arbor: Health Administration Press; 1993.

25. Holland W. Evaluation of health care. New York: Oxford University Press; 1983.

26. Long AF. Health services performance. New Hampshire: Groom Helm; 1985.

27. Glover JA. The Incidence of Tonsillectomy in School Children: (Section of Epidemiology and State Medicine). Proc R Soc Med 1938; 31:1219-36.

28. Reinhardt UE, Hussey PS, Anderson GF. Crossnational comparisons of health systems using OECD data, 1999. Health Aff (Millwood) 2002; 21:169-81.

29. Donabedian A. Evaluating the quality of medical care. Milbank Mem Fund Q 1966; 44:166206.

30. Donabedian A. The definition of quality and approaches to its assessment. Ann Arbor: Health Administration Press; 1980.

31. Cochrane AL. Effectiveness and efficiency: random reflections on health services. London: Nuffield Provincial Hospitals Trust; 1972.

32. Roemer MI. Disponibilidad de camas y utilización de los hospitales: un experimento natural. In: White K, Frenk J, Ordónez C, Paganini JM, Starfield B, editors. Investigaciones sobre servicios de salud: una antología. Washington DC: Organización Panamericana de la Salud; 1992. p. 231-9. (Publicación Científica, 534).

33. Hart JT. The inverse care law. Lancet 1971; 297:405-12.

34. Abel-Smith B. Value for money in Health Services. London: Heinemann; 1983.

35. Eisenberg JM. Physician utilization: the state of research about physicians' practice patterns. Med Care 1985; 23:461-83. 
36. Andersen R, Newman JF. Societal and individual determinants of medical care utilization in the United States. Milbank Mem Fund Q Health Soc 1973; 51:95-124.

37. Luft HS. Hospital volume, physician volume, and patient outcomes: assessing the evidence. Ann Arbor: Health Administration Press; 1990.

38. Drummond MF. Como avaliar uma política de saúde? Divulg Saúde Debate 1991; 3:26-41.

39. Banta HD. Tecnologia para a saúde. Divulg Saúde Debate 1991; 3:8-16.

40. Mooney GH. Economics, medicine and health care. 3rd Ed. New Jersey: FT PrenticeHall; 2003.

41. Chassin MR, Loeb JM, Schmaltz SP, Wachter RM. Accountability measures: using measurement to promote quality improvement. N Engl J Med 2010; 363:683-8.

42. Chassin MR. Improving the quality of health care: what's taking so long? Health Aff (Millwood) 2013 ; 32:1761-5.

43. Wennberg J, Gittelsohn A. Small area variations in health care delivery: a population-based health information system can guide planning and regulatory decision-making. Science 1973; 182:1102-8.

44. Wennberg JE. Tracking medicine a researcher's quest to understand health care. New York: Oxford University Press; 2010.

45. Brook RH, Davies-Avery A, Greenfield S, Harris LJ, Lelah T, Solomon NE, et al. Assessing the quality of medical care using outcome measures: an overview of the methods. Med Care 1977; 15(9 Suppl):1-165.

46. Relman AS. Assessment and accountability. N Engl J Med 1988; 319:1220-2.

47. Wyzewianski L. Quality of care: past achievements and future challenges. Inquiry 1988; 25:13-22.

48. Gaus CR, Simpson L. Reinventing health services research. Inquiry 1995; 32:130-4.

49. Marshall MN, Shekelle PG, Davies HT, Smith PC. Public reporting on quality in the United States and the United Kingdom. Health Aff (Millwood) 2003; 22:134-48.

50. Mohammed MA, Lilford R, Rudge G, Holder $\mathrm{R}$, Stevens A. The findings of the Mid-Staffordshire Inquiry do not uphold the use of hospital standardized mortality ratios as a screening test for 'bad' hospitals. QJM 2013; 106:849-54.

51. Vuori H. Quality assurance of health services. Copenhagen: World Health Organization; 1982.

52. Donabedian A. An introduction to quality assurance in health care. New York: Oxford University Press; 2003.

53. Black N. Patient reported outcome measures could help transform healthcare. BMJ 2013; 346:f167.

54. White KL, Frenk J, Ordonez C, Paganini JM, Starfield B editors. Investigaciones sobre servicios de salud: una antología. Washington DC: Pan American Health Organization; 1992. (Publicación Científica 534).

55. Clancy CM, Eisenberg JM. Outcomes research: measuring the end results of health care. Science 1998; 9:245-6.
56. Kohn LT, Corrigan JM, Donaldson MS; Committee on Quality of Health Care in America, Institute of Medicine. To err is human: building a safer health system. Washington DC: National Academy Press; 2000.

57. Eijkenaar F, Emmert M, Scheppach M, Schöffski O. Effects of pay for performance in health care: a systematic review of systematic reviews. Health Policy 2013; 110:115-30.

58. Margolis P, Provost LP, Schoettker PJ, Britto MT. Quality improvement, clinical research, and quality improvement research - opportunities for integration. Pediatr Clin N Am 2009; 56:831-41.

59. Marshall M, Provonost P, Dixon-Woods M. Promotion of improvement as a science. Lancet 2013; 381:419-21.

60. Davidoff F, Dixon-Woods M, Leviton L, Michie S. Demystifying theory and its use in improvement. BMJ Qual Saf 2015; 24:228-38.

61. Portela MC, Pronovost PJ, Woodcock T, Carter P, Dixon-Woods M. How to study improvement interventions: a brief overview of possible study types. BMJ Qual Saf 2015; 24:325-36.

62. Korenstein D, Falk R, Howell EA, Bishop T, Keyhani S. Overuse of health care services in the United States: an understudied problem. Arch Intern Med 2012; 172:171-8.

63. Keyhani S, Falk R, Bishop T, Howell E, Korenstein $\mathrm{D}$. The relationship between geographic variations and overuse of healthcare services: a systematic review. Med Care 2012; 50:257-261.

64. Leape LL. Scope of problem and history of patient safety. Obstet Gynecol Clin North Am 2008; 35:1-10.

65. Organisation for Economic Co-operation and Development. Geographic variations in health care: what do we know and what can be done to improve health system performance? OECD Health Policy Studies. Paris: OECD Publishing; 2014.

66. Glasziou P, Moynihan R, Richards T, Godlee F. Too much medicine; too little care. BMJ 2013; 347:f424.

67. Malhotra A, Maughan D, Ansell J, Lehman R, Henderson A, Gray M, et al. Choosing Wisely in the UK: the Academy of Medical Royal Colleges' initiative to reduce the harms of too much medicine. BMJ 2015; 350:h2308.

68. Amalberti R, Vincent C. Managing risk in hazardous conditions: improvisation is not enough. BMJ Qual Saf 2020; 29:60-3.

69. Dixon-Woods M. How to improve healthcare improvement - an essay by Mary DixonWoods. BMJ 2019; 366:15514.

70. Evans RG. The dog in the night-time: medical practice variations and health policy. In: Andersen TF, Mooney GH, editors. The challenges of medical practice variations. London: Macmillan International Higher Education; 1990; p. 117-52.

71. Kupersmith J, Eisen S. A new approach to health services research. Arch Intern Med 2012; 172:1033-4. 
72. Organização Pan Americana da Saúde. Rede de Investigação em Sistemas e Serviços de Saúde no Cone Sul (RED ISS). https://www.paho. org/bra/index.php?option=com_content \&vie $\mathrm{w}=$ article \&id $=542$ :rede-de-investigacao-emsistemas-e-servicos-de-saude-no-cone-sul-re $\mathrm{d}$-iss\&Itemid $=875$ (acessado em 03/01/2020).

73. Yazlle Rocha JS, Ortiz PC, Yang TF. La incidencia de cesáreas y la remuneración de la asistencia prestada durante el parto. In: White K, Frenk J, Ordónez C, Paganini JM, Starfield B, editors. Investigaciones sobre servicios de salud: una antología. Washington, DC: Pan American Health Organization; 1992. p. 1002-8. (Publicación Científica, 534).

74. Barros FC, Vaughan JP, Victoria CG. Por qué tanta cesáreas? Necesidad de mayores cambios de política en Brasil. In: White K, Frenk J, Ordónez C, Paganini JM, Starfield B, editors. Investigaciones sobre servicios de salud: una antología. Washington, DC: Pan American Health Organization; 1992. p. 1023-34. (Publicación Científica, 534).

75. Mello CG. A epidemiologia da cesariana. Rev Bras Hosp 1971; 3:29-33.

76. Vuori H. A qualidade da saúde. Divulg Saúde Debate 1991; 3:17-25.
77. Camacho LA, Hartz Z, Novaes HMD, Goldbaum M. [Editorial]. Cad Saúde Pública 1996; 12 Suppl 2:4-5.

78. Novaes HMD. Epidemiologia e avaliação em serviços de atenção médica: novas tendências na pesquisa. Cad Saúde Pública 1996; 12 Suppl 2:7-12.

79. Travassos C, Novaes HMD. Investigação e avaliação em serviços de saúde. Cad Saúde Pública 2004; 20 Suppl 2:144.

80. Centro Brasileiro de Estudos de Saúde. Economia da Saúde. Divulg Saúde Debate 2007; (37).

81. Santos EM, Cardoso GCP, Oliveira EA, Rizzotto MLF. Apresentação. Saúde Debate 2017; 41 (número especial):10-3.

82. Travassos C, Viacava F, Laguardia J. Os suplementos saúde na Pesquisa Nacional por Amostra de Domicílios (PNAD) no Brasil. Rev Bras Epidemiol 2008;11:98-112.

83. Travassos C, Viacava F. Utilização e financiamento de serviços de saúde: dez anos de informação das PNAD. Ciênc Saúde Colet 2011; 16:3646.

84. Viacava F, Ugá MA, Porto S, Laguardia J, Moreira RS. Avaliação de desempenho de sistemas de saúde: um modelo de análise. Ciênc Saúde Colet 2012; 17:921-34.
Recebido em 15/Jan/2020

Versão final reapresentada em 10/Mar/2020

Aprovado em 12/Mar/2020 